\title{
Short-term intravenous antimicrobial prophylaxis for elective rectal cancer surgery: results of a prospective randomized non-inferiority trial
}

\author{
Keiichiro Ishibashi $\cdot$ Hideyuki Ishida $\cdot$ Kouki Kuwabara $\cdot$ Tomonori Ohsawa \\ Norimichi Okada $\cdot$ Masaru Yokoyama $\cdot$ Kensuke Kumamoto
}

Received: 19 September 2012/ Accepted: 4 March 2013/Published online: 29 August 2013

(C) The Author(s) 2013. This article is published with open access at Springerlink.com

\begin{abstract}
Purpose To investigate the non-inferiority of postoperative single-dose intravenous antimicrobial prophylaxis to multiple-dose intravenous antimicrobial prophylaxis in terms of the incidence of surgical site infections (SSIs) in patients undergoing elective rectal cancer surgery by a prospective randomized study.

Methods Patients undergoing elective surgery for rectal cancer were randomized to receive a single intravenous injection of flomoxef (group 1) or five additional doses (group 2) of flomoxef after the surgery. All the patients had received preoperative oral antibiotic prophylaxis (kanamycin and erythromycin) after mechanical cleansing within $24 \mathrm{~h}$ prior to surgery, and had received intravenous flomoxef during surgery.

Results A total of 279 patients (including 139 patients in group 1 and 140 in group 2) were enrolled in the study. The incidence of SSIs was $13.7 \%$ in group 1 and $13.6 \%$ in group 2 (difference [95\% confidence interval]: $-0.2 \%$ [ -0.9 to $0.7 \%])$.

Conclusion The incidence of SSIs was not significantly different in patients undergoing elective rectal surgery who were treated using a single dose of postoperative antibiotics compared to those treated using multiple-dose antibiotics when preoperative mechanical and chemical bowel preparations were employed.
\end{abstract}

K. Ishibashi $(\varangle) \cdot$ H. Ishida $\cdot$ K. Kuwabara · T. Ohsawa .

N. Okada $\cdot$ M. Yokoyama $\cdot$ K. Kumamoto

Department of Digestive Tract and General Surgery, Saitama

Medical Center, Saitama Medical University, 1981 Kamoda,

Kawagoe, Saitama 350-8550, Japan

e-mail: k_ishi@saitama-med.ac.jp
Keywords Rectal cancer - Antimicrobial prophylaxis - Surgical site infection

\section{Introduction}

To prevent surgical site infections (SSIs) in patients undergoing colorectal surgery, the guidelines of the Centers for Disease Control and Prevention (CDC) published in 1999 [1] recommend the administration of a brief course of chemical preparations after mechanical bowel cleansing and limited use of intravenous antimicrobial prophylaxis (within $24 \mathrm{~h}$ of surgery). The validity of using limited-dose (such as single-dose) intravenous antimicrobial prophylaxis was demonstrated by a meta-analysis that included 17 randomized controlled trials [2]. However, these randomized controlled trials (RCTs) involved subjects undergoing colon surgery alone or a mixture of subjects undergoing colon and rectal surgery, with a lower percentage of those undergoing rectal surgery (range 18.6-55.0\%). Of these trials, only three [3-5] reported the incidence of SSIs in patients undergoing rectal surgery. Although the results indicated that there was no significant difference between single-dose and multiple-dose intravenous antimicrobial prophylaxis in terms of the reduction in the incidence of SSIs, the number of cases in each group was too small, ranging from only 18 to 49 cases.

In comparison to surgery for colon cancer, surgery for rectal cancer involves more extensive procedures, such as abdomino-perineal resection and pelvic exenteration. In addition, lateral lymph node dissection is frequently performed for cases of lower rectal cancer in Japan [6]. Ostomy construction, preoperative radiation and a very low anastomosis are all associated with a prolonged duration of surgery, greater risk of bacterial contamination and a wider 
dead space [7-10]. Previous reports from Western countries have suggested that the incidence of SSIs might differ between colon and rectal surgery $[11,12]$. Two important reports from Japan [13, 14] demonstrated a higher incidence of SSIs following rectal surgery than following colon surgery, even after the inclusion of various types of surgeries in the analyses. Therefore, determining the usefulness of short-term intravenous antimicrobial prophylaxis specifically for reducing the incidence of SSIs in patients undergoing rectal surgery would be of interest.

We carried out a prospective randomized non-inferiority trial to evaluate the usefulness of short-term intravenous antimicrobial prophylaxis combined with preoperative chemical preparation (administration of an antibiotic(s)), in terms of its efficacy for reducing the incidence of SSIs, and to identify the risk factors for SSIs in patients undergoing elective rectal cancer surgery in Japan.

\section{Patients and methods}

This study was conducted with the approval of the ethics committee of Saitama Medical Center, Saitama Medical University. Written informed consent was obtained from each patient.

\section{Patients}

A total of 295 patients underwent elective resectional surgery for rectal cancer, including rectosigmoid cancer, at our institution between January 2003 and September 2011.

Preoperative and intraoperative procedures related to the development of SSIs

All of the patients were given kanamycin ( $3 \mathrm{~g} /$ day) and erythromycin ( $2.4 \mathrm{~g} /$ day $)$ orally in three divided doses after mechanical bowel cleansing, within $24 \mathrm{~h}$ prior to surgery, in accordance with the CDC guidelines [1]. The mechanical bowel preparation consisted of bowel lavage with $2 \mathrm{~L}$ of polyethylene glycol or $34 \mathrm{~g}$ of magnesium citrate. Thereafter, $1 \mathrm{~g}$ of flomoxef (FMOX), a second-generation cephalosporin, was administered by intravenous injection, with an additional dose administered when the duration of surgery exceeded $3 \mathrm{~h}$.

The surgical wounds were covered with surgical towels. A stapled anastomosis was routinely performed for anterior resections. The stump of the Hartmann's pouch was also closed with a stapler. A hand-sewn anastomosis was made for intersphincteric resections. Irrespective of the type of surgery performed, the abdominal cavity was washed with copious amounts (2-3 L) of saline before closure of the wounds, and a closed-suction drain (BLAKE $^{\circledR}$ silicone drains, Ethicon,
Johnson \& Johnson, Somerville, NJ, USA) was placed presacrally, brought out through a separate stab wound, and connected to a J-VAC ${ }^{\circledR}$ suction reservoir (Ethicon, Johnson \& Johnson, Somerville, NJ, USA). All gloves were changed after the abdominal cavity was washed. After the fascia was approximated with absorbable sutures, the incisional site of the abdominal wall was washed with $200 \mathrm{~mL}$ of saline before closure of the skin, which was approximated with a skin stapler. The perineal skin was approximated with 2-0 nonabsorbable sutures when abodomino-perineal resection or pelvic exenteration was performed.

\section{Randomization of the patients}

After surgery, the patients were assigned to one of the following two groups using sealed envelopes containing randomized sheets. The patients in group 1 were given only single-dose intravenous antimicrobial prophylaxis $1 \mathrm{~h}$ after completion of the surgery, while the patients in group 2 were given an additional 5 doses over 2 consecutive days. Patients less than 20-year old, those with a known allergy to FMOX and those with any infection diagnosed within the previous 2 weeks, were excluded from this study.

\section{Postoperative follow-up}

The incision site was covered with a sterile dressing, which was removed within $48 \mathrm{~h}$ of surgery. In principle, the pelvic drain was removed within 5 days of surgery, and the staples were removed on postoperative day 7 . The wounds were inspected daily until the patients were discharged from the hospital, and each patient's wounds were inspected at the outpatient clinic 30 days after surgery. SSIs (incision site infections and organ/space infections) were recorded according to the definitions of the CDC [1]; however, no distinction was made between superficial and deep SSIs, because discrimination between the two was often difficult. Anastomotic dehiscence, which was classified into organ/ space infection, was confirmed by clinical and/or radiographic examinations. Remote infection was defined as an infection that occurred at a site other than the surgical site, such as pneumonia, enteritis, urinary tract infection or bloodstream (catheter-related) infection. The distribution of the location of the primary tumor and level of lymph node dissection was determined according to the guidelines of the Japanese Society for Cancer of the Rectum and Anus [15], and the distribution of the pathological stage was determined according to the TNM classification [16].

Sample size calculation

This trial was designed as a non-inferiority test to detect a $10 \%$ difference in the incidence of SSIs between the two 
groups with a confidence interval $(\mathrm{CI})$ of $95 \%$ and statistical power of $80 \%$, assuming that the incidence of SSIs in the multiple-dose intravenous antimicrobial prophylaxis group (group 2) would be $12 \%$, based on the our previous data on the incidence of SSIs after rectal cancer surgery performed between September 2000 and September 2001. Based on the above, it was calculated that a sample size of 131 would be required in each treatment arm. Then, the required number of patients was set at 140 per group, assuming a $10 \%$ potential dropout rate. [17].

\section{Statistical analysis}

The results in the single-dose prophylaxis group (group 1) were considered not to be inferior to those in the multipledose prophylaxis (group 2) if the lower limit of the twosided $95 \%$ CI for the difference in the incidence of SSIs was above $-10 \%$. The data are expressed as medians and ranges or $95 \% \mathrm{CI}$. For the statistical analyses, a statistical software package (StatView ver. 5.0, Abacus Concepts, Inc, Berkeley, CA, USA) running on a Windows personal computer was used. For the comparison of nominal variables, either the Chi-Square test or Fisher's exact probability test was used. For the comparison of continuous variables, Mann-Whitney's $U$ test was used. $P$ values of $<0.05$ were considered to denote statistical significance.

\section{Results}

Eligible patients

A flow chart of the randomization of the patients is shown in Fig. 1. Among the 295 patients who underwent elective surgery for rectal cancer during the specified period, a total of 16 patients were excluded, owing to refusal to participate in the study (two patients), inappropriate bowel preparation and/or fecal contamination in the surgical field (11 patients), or protocol violation with respect to the duration of antimicrobial prophylaxis after randomization (three patients, including two from group 1 and one from group 2). Thus, a total of 279 patients ( $n=139$ for group 1 and $n=140$ for group 2) were finally enrolled in the study.

\section{Patient characteristics}

The two groups did not differ significantly with respect to the sex ratio, age, ASA (American Society of Anesthesiologists physical status) score, BMI (body mass index), prevalence of diabetes mellitus, distribution of the location of the primary tumor, type of surgery, frequency of stoma creation, frequency of lateral lymph node dissection, frequency of combined resection of other organ(s), curative intent of surgery, duration of surgery, blood loss, level of lymph node dissection or distribution of the pathological stage (Table 1).

\section{SSIs}

The incidence of incision site infections was $5.0 \%$ (seven patients) in group 1 and $7.1 \%$ (10 patients) in group 2. All the incision site infections were considered to be superficial incision site infections. The incidence of organ/space infections was $10.8 \%$ (15 patients) in group 1 and $8.6 \%$ (12 patients) in group 2. Of the 27 organ/space infections, 12 (five in group 1 and seven in group 2) were related to anastomotic dehiscence. Three patients in group 1 and three patients in group 2 developed both incision site and organ/space infections. Therefore, the overall incidence of SSIs was $13.7 \%$ (19 patients) in group 1 and $13.6 \%$ (19 patients) in group 2 . The difference $[95 \% \mathrm{CI}]$ in the incidence of SSIs between the two groups was $-0.2 \%$ [ -0.9 to $0.7 \%$ ]. Because the lower limit of the two-sided $95 \% \mathrm{CI}$ was above $-10 \%$, the outcome in terms of the incidence of SSI in group 1 was considered to be noninferior to that in group 2 (Table 2).

\section{Subset analysis for SSIs}

A subset analysis was performed based on the types of surgery, namely, surgeries associated with a wide pelvic dead space (abdomino-pelvic resection and pelvic exenteration) vs. other surgeries, including anterior resection, intersphincteric resection, and Hartmann's procedure. In terms of the surgeries with a wide pelvic dead space, the overall incidence of SSIs ( 40.0 vs. $30.0 \%, P=0.51)$, incision site infections (5.0 vs. $20.0 \%, P=0.15)$ and organ/space infections ( 35.0 vs. $15.0 \%, P=0.14$ ) did not differ significantly between group 1 and group 2 . In terms of the surgeries, including anterior resection, intersphincteric resection and Hartmann's procedure, the overall incidence of SSIs (9.2 vs. $10.8 \%, P=0.68)$, incision site infections (5.0 vs. $5.0 \%, P>0.99)$ and organ/space infections (6.7 vs. $7.5 \%, P=0.82$ ) also did not significantly among between the groups (Table 3 ).

\section{Remote infections}

A remote infection was detected in nine $(6.5 \%)$ and 15 patients $(10.7 \%)$ in groups 1 and 2, respectively; there was no significant difference in the incidence of remote infection between the groups $(P=0.21)$ (Table 4$)$. Among the patients who developed enterocolitis, Clostridium difficile toxin A was detected in one patient from Group 1. This patient was treated with vancomycin and recovered without further complications. 
Fig. 1 A flow-chart of the randomization of the patients

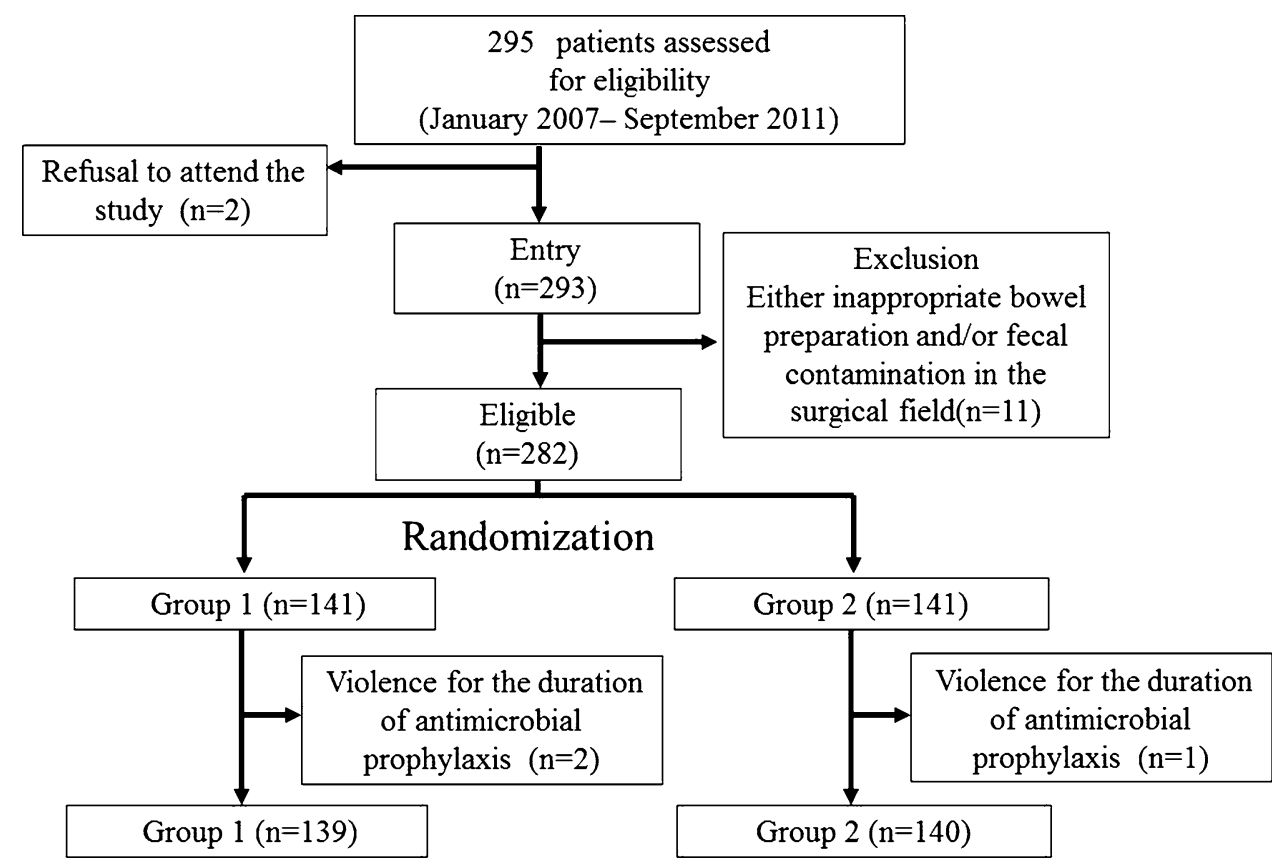

\section{Discussion}

This study clearly showed that, in patients administered a brief course of a chemical preparation after mechanical bowel cleansing prior to rectal cancer surgery, postoperative single-dose intravenous antimicrobial prophylaxis was non-inferior to multiple-dose prophylaxis in terms of the subsequent incidence of SSIs. Importantly, it appears that, irrespective of the type of rectal cancer surgery performed, selection between postoperative single-dose and multipledose intravenous antimicrobial prophylaxis in the immediate postoperative period may have little impact on the risk of the development of SSIs.

Even though this study was performed at a single institution, we believe that it had several distinct merits that enhanced its quality. For example, there were no interhospital variations, and the surgical procedures and pre-, intra- and postoperative management protocols related to SSIs were well standardized. To the best of our knowledge, this is the first randomized study comparing postoperative single-dose vs. multiple-dose intravenous antimicrobial prophylaxis following a course of oral antibiotics and mechanical bowel preparation prior to elective rectal cancer surgery, while Suzuki et al. [18] and our own group [19] have previously reported the non-inferiority of singledose to multiple-dose intravenous antimicrobial prophylaxis in elective colon cancer surgery performed after preoperative chemical and mechanical preparation.

The National Nosocomial Infections Surveillance (NNIS) system categorizes all colorectal surgeries into the same "COLO" group, and the incidence of SSIs within this group is stratified according to the NNIS risk index calculated based on the following three factors: the ASA score, wound classification and duration of operation (with $3 \mathrm{~h}$ set as the cut-off point) [20]. The NNIS risk index has been criticized as being unsuitable for risk evaluation in patients undergoing elective colorectal surgery, because most patients undergoing colorectal surgery have an ASA score of 1 or 2 and a clean-contaminated wound [10, 21, 22]. In terms of the incidence of incisional site infections, a retrospective analysis by Konishi et al. [13] demonstrated that the incidence in patients undergoing elective rectal surgery was $18.0 \%$, nearly twice as high as the incidence of $9.4 \%$ in patients undergoing elective colon surgery, although they did not evaluate organ/space infections. The Japanese Nosocomial Infection Surveillance (JNIS) system analyzed the incidence of SSIs after elective and emergency colon surgery and rectal surgery separately in 2009, and reported that the incidence of SSIs was $12.7 \%$ after colon surgery and $16.3 \%$ after rectal surgery [23]. It was therefore unclear whether the same perioperative management strategies, including the duration of intravenous antimicrobial prophylaxis, would be useful for both rectal and colon surgery.

In our present study, none of the patients had received preoperative chemoradiotherapy. In Western countries, chemoradiation prior to surgery is a standard treatment for locally advanced rectal cancer [24]. In Japan, surgery without neoadjuvant treatment, occasionally with lateral lymph node dissection, is the standard treatment for lower rectal cancer [6], even though (chemo) radiation for both upper and lower rectal cancers is performed in some institutions. A large randomized controlled study comparing total mesorectal excision (TME) with and without 
Table 1 Patient characteristics

\begin{tabular}{|c|c|c|c|}
\hline & Group $1(n=139)$ & Group $2(n=140)$ & $P$ value \\
\hline Sex (male:female) & $88: 51$ & $92: 48$ & 0.18 \\
\hline Age (years) ${ }^{a}$ & $65(35-90)$ & $65(33-91)$ & 0.81 \\
\hline $\mathrm{ASA}^{\mathrm{b}}$ score (I/II:III) & $127: 12$ & $125: 15$ & 0.56 \\
\hline BMI (body mass index) $)^{a}\left(\mathrm{~kg} / \mathrm{m}^{2}\right)$ & $22.0(15.6-32.0)$ & $22.4(13.2-33.0)$ & 0.57 \\
\hline Diabetes mellitus (present:absent) & $16: 123$ & $15: 125$ & 0.83 \\
\hline Location of the primary tumor & & & 0.18 \\
\hline Rectosigmoid & 46 & 38 & \\
\hline Upper rectum & 33 & 47 & \\
\hline Lower rectum & 60 & 55 & \\
\hline Type of surgery & & & 0.99 \\
\hline Hartmann's procedure & 4 & 5 & \\
\hline Anterior resection & 110 & 109 & \\
\hline Intersphincteric resection (ISR) & 5 & 6 & \\
\hline Abdomino-perineal resection & 18 & 19 & \\
\hline Pelvic exenteration & 2 & 1 & \\
\hline Total pelvic exenteration & 1 & 1 & \\
\hline Posterior (anterior) pelvic exenteration & 1 & 1 & \\
\hline Stoma creation $(\%)$ & $44(31.7 \%)$ & $47(33.6 \%)$ & 0.73 \\
\hline Colostomy $(\%)$ & $25(18.0 \%)$ & $24(17.1 \%)$ & \\
\hline Ileosomy $(\%)$ & $19(13.7 \%)$ & $23(16.4 \%)$ & \\
\hline Lateral lymph node dissection performed (\%) & $39(28.1 \%)$ & $30(21.4 \%)$ & 0.20 \\
\hline Combined resection of other organ(s) & $19(13.7 \%)$ & $31(22.9 \%)$ & 0.07 \\
\hline Liver & 4 & 8 & \\
\hline Small intestine or colon & 1 & 2 & \\
\hline Urinary bladder & 4 & 6 & \\
\hline Others & 11 & 17 & \\
\hline Curative intent of surgery ${ }^{c}$ & & & 0.12 \\
\hline R0 & 120 & 129 & \\
\hline $\mathrm{R} 1 / \mathrm{R} 2$ & 19 & 11 & \\
\hline Duration of surgery $(\min )^{\mathrm{a}}$ & $187(83-670)$ & $185(80-495)$ & 0.75 \\
\hline Blood loss $(\mathrm{g})^{\mathrm{a}}$ & $260(10-5850)$ & $310(10-3120)$ & 0.39 \\
\hline Lymph node dissection $^{\mathrm{d}}$ & & & 0.93 \\
\hline D0/D1 & 6 & 6 & \\
\hline D2 & 18 & 16 & \\
\hline D3 & 115 & 118 & \\
\hline pTNM stage ${ }^{c}$ & & & 0.11 \\
\hline Stage $0 / \mathrm{I} / \mathrm{II}$ & 65 & 79 & \\
\hline Stage III/IV & 74 & 61 & \\
\hline
\end{tabular}

a Median (range)

b American Society of Anesthesiologists physical status

c According to the 7th TNM Classification

d According to the Japaneses Classification of Colorectal

Carcinoma
There were two important randomized trials [26, 27] from Japan that have compared the risks of SSIs following single-dose intravenous antimicrobial prophylaxis [26] and preoperative chemical preparation [27] in patients undergoing elective colorectal surgery. Fujita et al. [26] reported that single-dose intravenous prophylaxis was associated with a $>3$-fold increase in the overall incidence of SSIs (14.3 vs. $4.3 \%$ ) compared to triple-dose intravenous antimicrobial prophylaxis in patients undergoing elective colorectal cancer surgery who had undergone mechanical cleansing, but not chemical preparation, preoperatively. 
Table 2 Surgical site infections

\begin{tabular}{lccc}
\hline & Group 1 $(n=139)$ & Group 2 $(n=140)$ & Difference $(95 \%$ CI $)$ \\
\hline Overall surgical site infections & $19(13.7 \%)$ & $19(13.6 \%)$ & $-0.2 \%(-0.9$ to $0.7 \%)$ \\
Incisional site infections & $7(5.0 \%)$ & $10(7.1 \%)$ & $12(8.6 \%)$ \\
Organ/space infections & $15(10.8 \%)$ & & \\
\hline
\end{tabular}

Table 3 Surgical site infections according to the type of surgery

\begin{tabular}{|c|c|c|c|c|c|c|c|c|c|}
\hline & \multicolumn{3}{|c|}{ Overall surgical site infections } & \multicolumn{3}{|c|}{ Incisional site infections } & \multicolumn{3}{|c|}{ Organ/space infections } \\
\hline & $\begin{array}{l}\text { Group 1 } \\
(n=139)\end{array}$ & $\begin{array}{l}\text { Group 2 } \\
(n=140)\end{array}$ & $P$ value & $\begin{array}{l}\text { Group 1 } \\
(n=139)\end{array}$ & $\begin{array}{l}\text { Group } 2 \\
(n=140)\end{array}$ & $P$ value & $\begin{array}{l}\text { Group 1 } \\
(n=139)\end{array}$ & $\begin{array}{l}\text { Group } 2 \\
(n=140)\end{array}$ & $P$ value \\
\hline $\begin{array}{l}\mathrm{APR} \text { or } \mathrm{PE} \\
\quad(n=40)\end{array}$ & $8 / 20(40.0 \%)$ & $6 / 20(30.0 \%)$ & 0.51 & $1 / 20(5.0 \%)$ & $4 / 20(20.0 \%)$ & 0.15 & $7 / 20(35.0 \%)$ & $3 / 20(15.0 \%)$ & 0.14 \\
\hline $\begin{array}{l}\mathrm{AR}, \mathrm{ISR} \text { or HA } \\
\quad(n=239)\end{array}$ & $11 / 119(9.2 \%)$ & $13 / 120(10.8 \%)$ & 0.68 & $6 / 119(5.0 \%)$ & $6 / 120(5.0 \%)$ & $>0.99$ & $8 / 119(6.7 \%)$ & $9 / 120(7.5 \%)$ & 0.82 \\
\hline
\end{tabular}

Table 4 Remote site infections

\begin{tabular}{llll}
\hline & $\begin{array}{l}\text { Group 1 } \\
(n=139)\end{array}$ & $\begin{array}{l}\text { Group 2 } \\
(n=140)\end{array}$ & $P$ value \\
\hline Remote site incisions & $9(6.5 \%)$ & $15(10.7 \%)$ & 0.21 \\
Pneumonia & 0 & 3 & \\
Enterocolitis & 3 & 4 & \\
Urinary tract infections & 4 & 6 & \\
Bloodstream infections & 2 & 2 & \\
\hline
\end{tabular}

They did not analyze the results separately in patients undergoing colon cancer surgery and rectal cancer surgery. Kobayashi et al. [27] reported that, in patients administered intravenous antimicrobial prophylaxis until postoperative day 3 , the use of the chemical preparation was not associated with a reduction in the overall incidence of SSIs; however, in a subset analysis, the use of the chemical preparation was associated with a significantly reduced incidence of SSIs in patients undergoing abdomino-perineal resection (58.8 vs. $11.1 \%$ ). This result was not consistent with our results demonstrating no significant differences in the incidence of SSIs after abdomino-perineal resection/pelvic exenteration between patients receiving single-dose and those receiving multiple-dose intravenous antimicrobial prophylaxis postoperatively $(P=0.74)$. These findings suggest that the risk of SSIs after abdomino-perineal resection might be influenced by the choice of chemical preparation and/or the duration of intravenous antimicrobial prophylaxis.

It has been reported that preoperative oral antibiotic use can induce Clostridium difficile-related colitis [28]. In our study, we detected Clostridium difficile toxin A in only one patient, who recovered with only conservative therapy. Because we did not conduct routine testing for Clostridium difficile, the exact incidence of "subclinical" Clostridium difficile-related infection in our patients remains unknown. Further studies are warranted to clarify this issue; however, a recent retrospective multicenter study demonstrated that chemical preparation did not increase the risk of Clostridium difficile infection in patients undergoing elective colon surgery [29].

In summary, our results suggest that the incidence of SSIs in patients undergoing elective rectal surgery following single-dose postoperative use of antibiotics was not significantly different from that of those undergoing multiple-dose treatments as long as preoperative mechanical and chemical bowel preparations were employed. Therefore, the administration of a single-dose of postoperative antibiotics is a valid measure to prevent postoperative surgical site infections.

Acknowledgments The authors would like to thank Professor Tomoyuki Kawada, Nippon Medical School, for his valuable assistance with the statistical analysis.

Conflict of interest Keiichiro Ishibashi and co-authors have no conflicts of interest to disclose.

Open Access This article is distributed under the terms of the Creative Commons Attribution License which permits any use, distribution, and reproduction in any medium, provided the original author(s) and the source are credited.

\section{References}

1. Mangram AJ, Horan TC, Pearson ML, Silver LC, Jarvis WR. Guideline for prevention of surgical site infection, 1999. Infect Control Hosp Epidemiol. 1999;20:250-78.

2. Song F, Glenny AM. Antimicrobial prophylaxis in colorectal surgery: a systemic review of randomized controlled trials. Br J Surg. 1998;85:1232-41. 
3. Åberg C, Thore M. Single versus triple dose antimicrobial prophylaxis in elective abdominal surgery and the impact on bacterial ecology. J Hosp Infect. 1991;18:149-54.

4. Juul P, Klaaborg KE, Kronborg O. Single or multiple doses of metronidazole and ampicillin in elective colorectal surgery. A randomized trial. Dis Colon Rectum. 1987;30:526-8.

5. Göransson G, Nilsson-Ehle I, Olsson SA, Petersson BG, Bengmark S. Single versus multiple dose doxycycline prophylaxis in elective colorectal surgery. Acta Chil Scand. 1984;150:245-9.

6. Watanabe T, Itabashi M, Shimada Y, Tanaka S, Ito Y, Ajioka Y, et al. Japanese Society for Cancer of the Colon and Rectum (JSCCR) guidelines 2010 for the treatment of colorectal cancer. Int J Clin Oncol. 2012;17:1-29.

7. Enker WE, Merchant N, Cohen AM, Lanouette NM, Swallow C, Guillem J, et al. Safety and efficacy of low anterior resection for rectal cancer. Ann Surg. 1999;230:544-54.

8. Law WL, Chu KW. Anterior resection for rectal cancer with mesorectal excision: a prospective evaluation of 622 patients. Ann Surg. 2004;240:260-8.

9. Sutton CD, Williams N, Marshall LJ, Lloyd G, Thomas WM. A technique for wound closure that minimizes sepsis after stoma closure. ANZ J Surg. 2002;72:766-7.

10. Tang R, Chen HH, Wang YL, Changchien CR, Chen JS, Hsu KC, et al. Risk factors for surgical site infection after elective resection of the colon and rectum: a single-center prospective study of 2809 consecutive patients. Ann Surg. 2001;234:181-9.

11. Nichols RL, Choe EU, Weldon CB. Mechanical and antibacterial bowel preparation in colon and rectal surgery. Chemotherapy. 2005;51:115-21.

12. Coppa GF, Eng K. Factors involved in antibiotic selection in elective colon and rectal surgery. Surgery. 1988;104:853-8.

13. Konishi T, Watanabe T, Kishimoto J, Nagawa H. Elective colon and rectal surgery differ in risk factors for wound infection. Results of prospective surveillance. Ann Surg. 2006;244:758-63.

14. Morikane K, Harihara Y, Konishi T. SSI surveillance for colorectal operations based on the JNIS system (in Japanese with English abstract). J Jpn Soc Surg Infect. 2007;4:129-34.

15. Japanese Society for Cancer of the Colon and Rectum. Japanese classification of colorectal carcinoma (Second English edition). Kanehara: Tokyo; 2009.

16. International Union Against Cancer (UICC) TNM classification of malignant tumours Seventh Edition (2009) In: Sobin LH, Gospodarowicz MK, Wittekind C, editors. New York: Wiley.

17. Makuch R, Simon R. Sample size requirements for evaluating a conservative therapy. Cancer Treat Rep. 1978;62:1037-40.

18. Suzuki T, Sadahiro S, Maeda Y, Yasuda S, Makuuchi H. The optimum duration of perioperative prophylactic antibiotic administration in patients with colon cancer: a randomized comparative study (in Japanese with English abstract). J Jpn Soc Surg Infect. 2006;3:267-71.

19. Ishibashi K, Kuwabara K, Ishiguro $T$, Ohsawa $T$, Okada $N$, Miyazaki $\mathrm{T}$, et al. Short-term intravenous antimicrobial prophylaxis in combination with preoperative oral antibiotics on surgical site infection and methicillin-resistant Staphylococcus aureus infection in elective colon cancer surgery: results of a prospective randomized trial. Surg Today. 2009;39:1032-9.

20. NNIS System. National Nosocomial Infections Surveillance (NNIS) system report, data summary from January 1992 through June 2004, issued October 2004. Am J Infect Control 2004; $32: 470-8$.

21. Smith RL, Bohl JK, McElearney ST, Friel CM, Barclay MM, Sawyer RG, et al. Wound infection after elective colorectal resection. Ann Surg. 2004;239:599-607.

22. Vandenbroucke-Grauls C, Schultsz C. Surveillance in infection control: are we making progress? Curr Opin Infect Dis. 2002; 15:415-9.

23. Kobayashi M, Takesue Y, Kitagawa Y, Kusunoki M, Sumiyama Y. Antimicrobial prophylaxis and colon preparation for colorectal surgery: results of a questionnaire survey of 721 certified institutions in Japan. Surg Today. 2011;41:1363-9.

24. National Comprehensive Cancer Network: Clinical Practice Guideline in Oncology, V3 2012, Rectal Cancer. http://www.ncc. org/. Accessed August 212012.

25. Marijnen CAM, Kapiteijn E, van de Velde CJH, Martijn H, Steup WH, Wiggers T, et al. Acute side effects and complications after short-term preoperative radiotherapy combined with total mesorectal excision in primary rectal cancer: report of a multicenter randomized trial. J Clin Oncol. 2002;20:817-25.

26. Fujita S, Saito N, Yamada T, Takii Y, Kondo K, Ohue M, et al. Randomized, multicenter trial of antibiotic prophylaxis in elective colorectal surgery. Arch Surg. 2007;142:657-61.

27. Kobayashi M, Mohri Y, Tonouchi H, Miki C, Nakai K, Kusunoki $\mathrm{M}$. Randomized clinical trial comparing intravenous antimicrobial prophylaxis alone with oral and intravenous antimicrobial prophylaxis for prevention of a surgical site infection in colorectal surgery. Surg Today. 2007;37:383-8.

28. Wren SM, Ahmed N, Jamal A, Safadi BY. Preoperative oral antibiotics in colorectal surgery increase the rate of Clostridium difficile colitis. Arch Surg. 2005;140:752-6.

29. Krapohl GL, Phillips LRS, Campbell DA Jr, Hendren S, Banerjee $\mathrm{M}$, Metzger B, et al. Bowel preparation for colectomy and risk of Clostrium difficile infection. Dis Colon Rectum. 2011;54:810-7. 